Preprint de "On Societies Choosing Social Outcomes, and their Memberships: Internal Stability and Consistency", Gustavo Bergantiños, Jordi Massó i Alejandro Neme. Theory and Decision, en prensa (2018). Lliurat a Springer el setembre de 2017 


\title{
On Societies Choosing Social Outcomes, and their
}

\section{Memberships: Internal Stability and Consistency*}

\author{
Gustavo Bergantiños ${ }^{\dagger}$, Jordi Massó $^{\ddagger}$ and Alejandro Neme ${ }^{\S}$
}

September 2017

\begin{abstract}
We consider a society whose members have to choose not only an outcome from a given set of outcomes but also a subset of agents that will remain members of the society. We study the extensions of approval voting, scoring methods and the Condorcet winner to our setting from the point of view of their internal stability and consistency properties.
\end{abstract}

Journal of Economic Literature Classification Number: D71.

Keywords: Internal Stability; Consistency; Efficiency; Neutrality; Anonymity.

${ }^{*}$ We are grateful to two referees for many useful comments and suggestions. The work of G. Bergantiños is partially supported by research grants ECO2014-52616-R from the Spanish Ministry of Science and Competitiveness, GRC 2015/014 from "Xunta de Galicia", and 19320/PI/14 from "Fundación Séneca de la Región de Murcia". J. Massó acknowledges financial support from the Spanish Ministry of Economy and Competitiveness, through the Severo Ochoa Programme for Centres of Excellence in R\&D (SEV-2015-0563) and grant ECO2014-53051, and from the Generalitat de Catalunya, through grant SGR2014-515. The paper was partly written while J. Massó was visiting the Department of Economics at Stanford University; he wishes to acknowledge its hospitality as well as financial support from the Ministerio de Educación, Cultura y Deporte through project PR2015-00408. The work of A. Neme is partially supported by the Universidad Nacional de San Luis, through grant 319502, and by the Consejo Nacional de Investigaciones Científicas y Técnicas (CONICET), through grant PIP 112-200801-00655.

${ }^{\dagger}$ Facultade de Económicas, Universidade de Vigo. 36310, Vigo (Pontevedra), Spain. E-mail: gbergant@uvigo.es

${ }^{\ddagger}$ Universitat Autònoma de Barcelona and Barcelona Graduate School of Economics. Departament d'Economia i d'Història Econòmica, Campus UAB, Edifici B. 08193, Bellaterra (Barcelona), Spain. E-mail: jordi.masso@uab.es

${ }^{\S}$ Instituto de Matemática Aplicada de San Luis. Universidad Nacional de San Luis and CONICET. Ejército de los Andes 950. 5700, San Luis, Argentina. E-mail: aneme@unsl.edu.ar 


\section{Introduction}

Classical social choice studies problems where a fixed set of agents have to choose an outcome from a given set of outcomes, and agents have preferences only over this set. However, there are settings where, depending on the chosen outcome, some agents might want to leave the society; and this, in turn, might be perceived by some agents that were initially willing to remain in the society as negative, and now they might also want to leave. For instance, in the case of an excludable and costly public good, agents' preferences may depend on the level of the public good and on the size of the set of agents consuming (and contributing to finance) it. Also, when membership is voluntary in a double sense: no agent can be forced to belong to the final society and any agent can be part of it, if the agent whishes to be. A prototypical example of this class of problems is a political party, whose membership may depend on the positions that the party takes on issues like the death penalty, abortion or the possibility of allowing a region of a country to become independent. A professor in a department may start looking for a position elsewhere if he considers that the recruitment of the department has not being satisfactory to his standards; and this, in turn might trigger further exits. To be able to deal with such situations the classical social choice model has to be modified to include explicitly the possibility that initial members of the society may leave it as the consequence of the chosen outcome and hence, preferences have to be extended to order pairs formed by the final society and the chosen outcome.

There is a large literature that has already considered explicitly the dependence of the final society on the choices made by the initial society. ${ }^{1}$ Barberà, Maschler and Shalev (2001), Barberà and Perea (2002), and Berga, Bergantiños, Massó and Neme (2004, 2006, 2007) study alternative models in terms of the voting methods used to choose the outcome and the timing under which members reconsider their membership. Jackson and Nicolò (2004) study the provision of excludable public goods when agents care also about the number of other consumers. In this note (as we also do in the companion paper Bergantiños, Massó, and Neme (2017)), we look at the general setting without being specific about the two issues. We do that by considering that the set of alternatives are all pairs formed by a subset of the original society $N$ (an element in $2^{N}$, the subset of agents that will remain in the society) and an outcome in

\footnotetext{
${ }^{1}$ See for instance Roberts (1999) for problems related to club formation and Sobel (2000) for the declining of standards in societies that choose their members.
} 
$X$. Then, we assume that agents' preferences are defined over the set of alternatives $2^{N} \times X$ and satisfy two natural requirements. First, each agent has a strict preference between any two alternatives, provided he belongs to at least one of the two corresponding societies. Second, each agent is indifferent between any two alternatives, provided he is not a member of any of the two corresponding societies; namely, agents that do not belong to the final society do not care about neither its composition nor the chosen outcome.

We consider rules that operate on this restricted domain of preference profiles by selecting, for each profile, an alternative (a final society and an outcome). In Bergantiños, Massó, and Neme (2017) we characterize the class of strategy-proof, unanimous and outsider independent rules as the family of all serial dictator rules. ${ }^{2}$

For applications where the profile is common knowledge (and hence, the strategic revelation of agents' preferences is not an issue) we focus on internally stable and consistent rules. ${ }^{3}$ Internal stability says that nobody can force an agent to remain in the society if the agent does not want to do so. This is a minimal requirement of individual rationality, and it is a desirable property whenever membership is voluntary. A rule is consistent if the following property holds. Apply the rule to a given profile and consider the new problem where the new society is formed by the subset of agents chosen at the original profile. A consistent rule has to choose, at the subprofile of preferences of the agents that remain in the society, the same alternative. Thus, a consistent rule does not have to be reapplied after an alternative has been chosen. We want to emphasize that, in contrast with the standard notion, our consistency property requires to re-apply the rule only to the (non-empty) set of agents that has been selected at the original profile. We think that this is the relevant consistency notion because the new composition of the society is not just a hypothetical circumstance, it is a fact. Internal stability and consistency are desirable if we want to interpret the alternative chosen by the rule as being the final one, in a double sense. Members of the final society want to stay and if the rule would be applied again to the final society it would choose the same final society and the same outcome, so there is no need to do so.

\footnotetext{
${ }^{2} \mathrm{~A}$ rule is outsider independent if it is invariant with respect to the change of the preferences of an agent who is not a member of the two final societies.

${ }^{3}$ For the study of consistent rules in other social choice settings see, for instance, Sasaki and Toda (1992), Thomson (1994, 2007), Özkal-Sanver (2013), Nizamogullari and Özkal-Sanver (2014, 2015) and Bergantiños, Massó and Neme (2015).
} 
We adapt well-known voting methods to our setting with the goal of making them either internally stable or consistent, or both. ${ }^{4}$ We show that two prominent scoring methods, plurality voting and the Borda rule, do not satisfy consistency. However, approval voting not only satisfies internal stability and consistency but it also satisfies efficiency and neutrality. Finally, we show that the Condorcet winner is internally stable, consistent, efficient, neutral and anonymous at those profiles where an alternative beats all other alternatives by majority voting (namely, whenever it is a well-defined rule).

The paper is organized as follows. In Section 2 we describe the model. Section 3 contains the definitions of the properties of rules that we are interested in. Section 4 contains the analysis of well-known rules from the point of view of their internal stability and consistency properties. Section 5 has two final remarks.

\section{Preliminaries}

This section follows closely Bergantiños, Massó, and Neme (2017). Let $N=\{1, \ldots, n\}$, with $n \geq 2$, be the set of agents who have to choose an outcome from a given finite set $X$ of possible outcomes. We are interested in situations where some agents may not be part of the final society, perhaps as the consequence of the chosen outcome. To model such situations, let $A=2^{N} \times X$ be the set of alternatives and assume that each $i \in N$ has preferences over $A$. Observe that for all $x \in X,(\varnothing, x) \in A$; so we are admitting the possibility that the final society does not have any member. We will often use the notation $a$ for a generic alternative $(S, x) \in A$; i.e., $a \equiv(S, x), a^{\prime} \equiv\left(S^{\prime}, x^{\prime}\right)$, and so on. Let $R_{i}$ denote $i$ 's (weak) preference over $A$, where for any pair $a, a^{\prime} \in A, a R_{i} a^{\prime}$ means that $i$ considers $a$ to be at least as good as $a^{\prime}$. Let $P_{i}$ and $I_{i}$ denote the strict and indifference relations over $A$ induced by $R_{i}$, respectively; namely, for any pair $a, a^{\prime} \in A, a P_{i} a^{\prime}$ if and only if $a R_{i} a^{\prime}$ and $\neg a^{\prime} R_{i} a$, and $a I_{i} a^{\prime}$ if and only if $a R_{i} a^{\prime}$ and $a^{\prime} R_{i} a$. We assume that each $i$ does not care about any of those alternatives at which $i$ does not belong to their corresponding final societies. Besides $i$ is not indifferent between any pair of alternatives at which $i$ belongs to at least one of the two corresponding final societies. Namely, we assume that $i$ 's preferences $R_{i}$ satisfy the following two properties: for all $S, T \in 2^{N}$ and $x, y \in X$,

\footnotetext{
${ }^{4}$ Example 1 shows that without those adaptations, the voting methods are not internally stable.
} 
(P.1) if $i \notin S \cup T$ then $(S, x) I_{i}(T, y)$; and

(P.2) if $i \in S \cup T$ and $(S, x) \neq(T, y)$ then either $(S, x) P_{i}(T, y)$ or $(T, y) P_{i}(S, x)$.

The fact that agents' preferences satisfy (P.1) is the reason why our model cannot mechanically be embedded into the classical model. A specific analysis is required, partly because properties like internal stability and consistency become specially meaningful under this domain restriction. We see property (P.1) as being a natural assumption for our setting, and it is a critical requirement for our results to hold. Let $\mathcal{R}_{i}$ be the set of preferences of $i$ satisfying (P.1) and (P.2), and let $\mathcal{R}=\times_{i \in N} \mathcal{R}_{i}$ be the set of (preference) profiles.

We denote the subset of alternatives with the property that $i$ is not a member of the corresponding final society by $[\varnothing]_{i}=\{(S, x) \in A \mid i \notin S\}$. By (P.1), $i$ is indifferent among them; i.e.,

$$
[\varnothing]_{i}=\left\{a \in A \mid a I_{i}(\varnothing, x) \text { for some } x \in X\right\} .
$$

By (P.1), $(\varnothing, x) I_{i}(\varnothing, y)$ for all $x, y \in X$ and $[\varnothing]_{i}$ can be seen as the indifference class generated by the empty society. Observe that $[\varnothing]_{i}$ may be at the top of $i$ 's preferences. With an abuse of notation we often treat, when listing a preference ordering, the indifference class $[\varnothing]_{i}$ as if it were an alternative; for instance, given $R_{i}$ and $a \in A$ we write $a R_{i}[\varnothing]_{i}$ to represent that $a R_{i} a^{\prime}$ for all $a^{\prime} \in[\varnothing]_{i}$.

The top of $R_{i}$, denoted by $\tau\left(R_{i}\right)$, is the set of all best alternatives according to $R_{i}$; namely,

$$
\tau\left(R_{i}\right)=\left\{a \in A \mid a R_{i} a^{\prime} \text { for all } a^{\prime} \in A\right\} .
$$

Note that by (P.1) and (P.2), $\tau\left(R_{i}\right)$ is either a singleton set or the indifference class $[\varnothing]_{i}$.

A rule is a social choice function $f: \mathcal{R} \rightarrow A$ selecting, for each profile $R \in \mathcal{R}$, an alternative $f(R) \in A$. To be explicit about the two components of the alternative chosen by $f$ at $R$, we will often write $f(R)$ as $\left(f_{N}(R), f_{X}(R)\right)$, where $f_{N}(R) \in 2^{N}$ and $f_{X}(R) \in X$.

To clarify the model, we relate it with two of the examples used in the introduction. The set of agents $N$ corresponds to the initial members of the political party, the set of outcomes $X$ to the set of choices that the political party has to make and the set $S$, if the chosen alternative is $(S, x)$, to the set of final members of the party that stay after it supports outcome $x$. Similarly, $N$ corresponds to the set of professors in the department, the set of outcomes $X$ to all subsets of candidates and the set $S$, if the chosen alternative is $(S, x)$, to the set of professors who remain in the department after the subset of candidates $x$ has been hired. 


\section{Properties of rules}

In this section we present several properties that a rule may satisfy. The first two impose conditions at each profile.

A rule is efficient if it always selects a Pareto optimal allocation.

Efficiency For each $R \in \mathcal{R}$ there is no $a \in A$ with the property that $a R_{i} f(R)$ for all $i \in N$ and $a P_{j} f(R)$ for some $j \in N$.

The next property is related to the stability of a rule, and it captures the idea that agents are able to exit a society at their free will. Internal stability says that no agent belonging to the final society would prefer to leave it.

Internal stability For all $R \in \mathcal{R}$ and all $i \in f_{N}(R), f(R) P_{i}\left(f_{N}(R) \backslash\{i\}, f_{X}(R)\right)$.

It is immediate to see that internal stability is indeed equivalent to the requirement of individual rationality (for all agents); i.e., for all $R$ and all $i, f(R) R_{i}[\varnothing]_{i}$. Individual rationality implies internal stability by their definitions and (P.2). Assume $f$ is internally stable and let $R$ be arbitrary. If $i \in f_{N}(R)$ then $f(R) P_{i}[\varnothing]_{i}$. If $i \notin f_{N}(R)$ then, by (P.1), $f(R) I_{i} a$ for any $a \in[\varnothing]_{i}$. Thus, for all $i, f(R) R_{i}[\varnothing]_{i} .^{5}$

The next three properties impose conditions by comparing the alternatives chosen by the rule at two different profiles. A rule is anonymous if the names of the agents are not relevant to select the alternative. To define it formally, let $\pi: N \rightarrow\{1, \ldots, n\}$ be an ordering of $N$ (i.e., a one-to-one mapping). Given $i \in N, \pi(i)$ (or $\pi_{i}$ to simplify notation) is the agent assigned to $i$ after applying $\pi$ to $N$. The set of all orderings $\pi: N \rightarrow\{1, \ldots, n\}$ will be denoted by П. Given $S \in 2^{N}$ and $\pi \in \Pi$ we denote by $\pi(S)$ the subset of agents associated to $S$ by $\pi$; namely, $\pi(S)=\{i \in N \mid \pi(j)=i$ for some $j \in S\}$. Given $R \in \mathcal{R}$ and $\pi \in \Pi$ we denote by $R^{\pi}$ the new profile where, for all $i \in N$, agent $\pi(i)$ has the preference obtained from $R_{i}$ after replacing each $(S, x)$ by $(\pi(S), x)$.

Anonymity For all $R \in \mathcal{R}$ and all $\pi \in \Pi, f\left(R^{\pi}\right)=\left(\pi\left(f_{N}(R)\right), f_{X}(R)\right)$.

A rule is neutral if the names of the outcomes do not play any role in selecting the social alternative. To define it formally, let $\sigma: X \rightarrow X$ be a permutation of $X$. Given $x \in X, \sigma(x)$

\footnotetext{
${ }^{5}$ We will later argue (at the beginning of Section 4) that serial dictator rules, as defined in Bergantiños, Massó and Neme (2017), are not internally stable.
} 
is the outcome assigned to $x$ after applying $\sigma$ to $X$. The set of all permutations $\sigma: X \rightarrow X$ will be denoted by $\Sigma$. Let $Y \subseteq X$ be non-empty and $\sigma \in \Sigma$. Denote by $\sigma(Y)$ the subset of outcomes associated to $Y$ by $\sigma$; namely, $\sigma(Y)=\{x \in X \mid \sigma(y)=x$ for some $y \in Y\}$. Given $R \in \mathcal{R}$ and $\sigma \in \Sigma$ we denote by $R^{\sigma}$ the profile where, for all $i \in N$, the preference $R_{i}^{\sigma}$ is obtained from $R_{i}$ after replacing each $(S, x)$ by $(S, \sigma(x))$.

Neutrality For all $R \in \mathcal{R}$ and all $\sigma \in \Sigma, f\left(R^{\sigma}\right)=\left(f_{N}(R), \sigma\left(f_{X}(R)\right)\right)$.

A rule is consistent if the following requirement holds. Apply the rule to a given profile and consider the subset of agents that are members of the final society. Construct the new subprofile of preferences restricted to this new set of chosen agents. Then, the rule does not require to modify the chosen alternative because if it were applied to the new subprofile the new alternative would coincide with the alternative chosen at the original profile. To define the property formally, we first need additional notation. Given $R \in \mathcal{R}$ and $S \subset N$, denote by $R_{\mid S}=\left(\left(R_{\mid S}\right)_{i}\right)_{i \in S}$ the restriction of $R$ to $2^{S} \times X$. Namely, given $i \in S, T \cup T^{\prime} \subset S$ and $x, y \in X$, $(T, x)\left(R_{\mid S}\right)_{i}\left(T^{\prime}, y\right)$ if and only if $(T, x) R_{i}\left(T^{\prime}, y\right)$. Second, we specify how a given rule $f$ can be applied to a subprofile by considering it as it were a family of rules, one for each non-empty subset of $N$. Given $S \in 2^{N} \backslash\{\varnothing\}$ denote by $\mathcal{R}^{S}$ the set of subprofiles $R_{\mid S}=\left(\left(R_{\mid S}\right)_{i}\right)_{i \in S}$. Thus, a rule $f$ can be identified with the collection $\left\{f^{S}\right\}_{S \in 2^{N} \backslash\{\varnothing\}}$ of rules where for each $S \in 2^{N} \backslash\{\varnothing\}$, $f^{S}: \mathcal{R}^{S} \rightarrow 2^{S} \times X$. We often omit the superscript $S$ and write $f\left(R_{\mid S}\right)$.

Consistency For all $R \in \mathcal{R}, f(R)=f\left(R_{\mid f_{N}(R)}\right)$ whenever $f_{N}(R) \neq \varnothing$.

In contrast with the standard notion, and as we have already said in the Introduction, our consistency property requires to re-apply the rule only to the (non-empty) set of agents that has been selected at the original profile. Hence, our notion is weaker since the choice of the rule $f(R)$ is re-evaluated only at the profile $R_{\mid f_{N}(R)}$, instead of at all $R_{\mid S}$ (for $S \subset N$ ). We think that this is the relevant consistency notion because the new composition of the society is not just a hypothetical circumstance, it is a fact. And indeed, the new set of agents might be willing to reconsider their membership and the chosen outcome; particularly because, in the choice of the later, preferences of members that are not anymore in the society may have played a relevant role. Consistency says that the original choice, if re-evaluated by the new society by means of the same rule, will continue to be chosen.

We say that a rule satisfies any of the above properties at $R$ if the condition defining the property holds at $R$. 


\section{Internally stable and consistent rules}

In Bergantiños, Massó and Neme (2017) we characterize the class of all strategy-proof, unanimous and outsider independent rules as the family of serial dictator rules. ${ }^{6}$ A serial dictator rule induced by $\pi \in \Pi$ and $x \in X$, denoted by $f^{\pi, x}$, proceeds (in up to $n$ steps) as follows. Fix a profile $R \in \mathcal{R}$ and look for any alternative $\left(S_{1}, x_{1}\right)$ in the best indifference class of agent $\pi_{1}$, the first agent in the ordering induced by $\pi$. If $\pi_{1} \in S_{1}$, set $f^{\pi, x}(R)=\left(S_{1}, x_{1}\right)$. Otherwise, look for any alternative $\left(S_{2}, x_{2}\right)$ in the best indifference class of agent $\pi_{2}$, the second agent in the ordering induced by $\pi$, only among those classes satisfying the property that $\pi_{1} \notin S_{2}$, If $\pi_{2} \in S_{2}$, set $f^{\pi, x}(R)=\left(S_{2}, x_{2}\right)$. Otherwise, proceed similarly until the $n$-th step, if reached, by looking for any alternative $\left(S_{n}, x_{n}\right)$ in the best indifference class of agent $\pi_{n}$, the last in the ordering induced by $\pi$, only among those classes satisfying the property that for each $k \in\{1, \ldots, n-1\}, \pi_{k} \notin S_{n}$. If $\pi_{n} \in S_{n}$, set $f^{\pi, x}(R)=\left(S_{n}, x_{n}\right)$. Otherwise, and since no agent wants to stay in the society whatever element of $X$ is selected, set $f^{\pi, x}(R)=(\varnothing, x)$. So, $x$ plays the role of the residual outcome only when no agent wants to stay in the society under any circumstance.

It is obvious to see that any serial dictator rule satisfies efficiency but fails anonymity and neutrality. Consider a problem where the top alternative for agent $\pi_{1}$ is $(N, x)$ but $(\varnothing, x) P_{\pi_{2}}(N, x)$. Since the serial dictator rule selects $(N, x)$, it does not satisfy internal stability. We now see that the serial dictator rule satisfies consistency. Assume that $f^{\pi, x}(R)=$ $(S, y)$. Let $\pi_{i}$ be the first agent in $S$ according with $\pi$. Notice that $(S, y)$ is the best alternative of agent $\pi_{i}$ in

$$
\left\{(T, z) \mid \pi_{j} \notin T \text { for all } j<i \text { and } z \in X\right\},
$$

which coincides with $f^{\pi_{\mid S}, x}\left(R_{\mid S}\right)$, where $\pi_{\mid S}$ is the restriction of $\pi$ into $S$.

Here, we consider situations where the strategic manipulation in the preference revelation game is not an issue and we will look for internally stable and consistent rules. To do so, we first ask whether two prominent procedures in classical social choice satisfy them. Recall that in the classical setting the goal is to select an outcome, from a given set $X$, taking into account (partially or fully) the strict preferences of agents over $X$. The procedures we consider are:

\footnotetext{
${ }^{6} \mathrm{~A}$ rule is unanimous if it always selects an alternative belonging to the set of common best alternatives, whenever this set is nonempty. A rule is outsider independent if it is invariant with respect to the change of preferences of an agent who is not a member of the two final societies.
} 
1. Approval voting. Each $i \in N$ votes for a subset $X_{i}$ of $X$. For each outcome $x \in X$, compute the number of votes received by $x$; namely, $\left|\left\{i \in N \mid x \in X_{i}\right\}\right|$. The outcome with more votes is selected. A tie-breaking rule should be applied whenever two or more outcomes obtain the largest number of votes. Note that approval voting is not a rule because $i$ 's vote $X_{i}$ is not completely determined by $P_{i}$

2. Scoring methods. Each $i \in N$ strictly ranks all outcomes. Assign to each outcome a pre-established decreasing number of points depending on its position in $i$ 's ranking. ${ }^{7}$ Compute the sum of the points obtained by each outcome. Select the one with more points. A tie-breaking rule should be applied whenever two or more outcomes obtain the largest number of points.

We tentatively adapt the two procedures to our setting to deal with the indifferences generated by (P.1) and to define approval voting as a proper rule.

1. Approval voting. Each $i \in N$ votes for all $a \in A$ such that $a P_{i}[\varnothing]_{i}$ (if any).

2. Scoring methods. For each $i \in N$, assign a pre-established decreasing number of points to each outcome depending on its position in $i$ 's ranking but considering $[\varnothing]_{i}$ as a single alternative. For each $(S, x) \in A$ and each $i \in N \backslash S$, assign to $(S, x)$ the score obtained by $[\varnothing]_{i}$.

Example 1 below shows that none of these extensions satisfy internal stability.

Example 1 Assume $n \geq 3$ and fix $x \in X$. Let $R \in \mathcal{R}$ be any profile such that $\tau\left(R_{1}\right)=[\varnothing]_{1}$ and for all $i \in N \backslash\{1\}, \tau\left(R_{i}\right)=(N, x)$ and $[\varnothing]_{i} P_{i}(S, y)$ for all $S \neq N, i \in S$ and $y \in X$. Then, each of the adapted procedures chooses $(N, x)$ at $R$. Nevertheless, $(N, x)$ is not internally stable because agent 1 prefers to leave the society.

Since we are interested in identifying rules satisfying internal stability, we modify the previous methods by considering only votes to alternatives $(S, x)$ that are internally stable for each $i \in S$ according to $R_{i}$; namely, only alternatives $(S, x)$ with the property that $(S, x) P_{i}[\varnothing]_{i}$

\footnotetext{
${ }^{7}$ The Borda rule is the scoring method when the points are the integers $k-1, \ldots, 1,0$ where $k$ is the number of alternatives. The Plurality rule is the scoring method when one point is assigned to the top-ranked alternative and zero points are assigned to the other alternatives.
} 
for each $i \in S$ can receive votes, not only from $i$ but also from all other agents (we call these alternatives unanimously internally stable). In approval voting each agent votes, among the set of alternatives at which he is a member of the society, only for those that are unanimously internally stable. If no alternative receives a vote the rule selects a particular alternative $(\varnothing, x)$ by a tie-breaking rule that will be described later. In a scoring method we consider only the rank, given agents' preferences, among the unanimously internally stable alternatives. ${ }^{8}$ Hence, at the profile of Example 1 each $i$ votes for $[\varnothing]_{i}$ and $(\varnothing, y)$ is selected according to some preestablished $y$. With these modifications, they satisfy internal stability by definition.

We now focus on two prominent scoring methods: plurality voting and the Borda rule, respectively denoted by $f^{P}$ and $f^{B}$. Our first result is negative: they do not satisfy consistency (independent of the rule used to break ties). To see that, consider Example 2 below.

Example 2 Let $N=\{1,2,3,4,5,6\}$ and $X=\left\{y_{1}, y_{2}, y_{3}, y_{4}, y_{5}\right\}$ be respectively the set of agents and outcomes and consider the following profile $R \in \mathcal{R}$. For each $i \in N,(S, x) P_{i}[\varnothing]_{i}$ whenever $i \in S$. Observe that for each $i \in N$, \#\{(S,x)|(S,x) $\left.P_{i}[\varnothing]_{i}\right\}=2^{5} \times 5=160$ is the number of alternatives that at $R$ each agent $i$ ranks strictly above $[\varnothing]_{i}$. In addition, $R$ is one among all profiles satisfying the following properties, where the first column indicates the rank of each of the six preference relations.

\begin{tabular}{c|cccccc} 
Rank & $R_{1}$ & $R_{2}$ & $R_{3}$ & $R_{4}$ & $R_{5}$ & $R_{6}$ \\
\hline 1 & $\left(N, y_{1}\right)$ & $\left(N, y_{2}\right)$ & $\left(N, y_{3}\right)$ & $\left(N \backslash\{6\}, y_{4}\right)$ & $\left(N \backslash\{6\}, y_{4}\right)$ & $\left(N, y_{5}\right)$ \\
2 & $\left(N \backslash\{6\}, y_{1}\right)$ & $\left(N \backslash\{6\}, y_{1}\right)$ & $\left(N \backslash\{6\}, y_{1}\right)$ & $\left(N \backslash\{1\}, y_{4}\right)$ & $\left(N \backslash\{1\}, y_{4}\right)$ & $\left(N, y_{4}\right)$ \\
3 & $\left(N \backslash\{6\}, y_{4}\right)$ & $\left(N \backslash\{6\}, y_{4}\right)$ & $\left(N \backslash\{6\}, y_{4}\right)$ & $\left(N \backslash\{2\}, y_{4}\right)$ & $\left(N \backslash\{2\}, y_{4}\right)$ & $\left(N, y_{3}\right)$ \\
4 & & & $\left(N \backslash\{3\}, y_{4}\right)$ & $\left(N \backslash\{3\}, y_{4}\right)$ & $\left(N, y_{2}\right)$ \\
5 & & & $\left(N \backslash\{6\}, y_{1}\right)$ & $\left(N \backslash\{6\}, y_{1}\right)$ & $\left(N, y_{1}\right)$ \\
$\vdots$ & $\vdots$ & & $\vdots$ & $\vdots$ & $\vdots$ \\
159 & $\left(N \backslash\{3\}, y_{4}\right)$ & $\left(N, y_{1}\right)$ & $\left(N, y_{2}\right)$ & $\left(N, y_{3}\right)$ & $\left(N, y_{5}\right)$ & $\left(N, y_{5}\right)$ \\
160 & $\left(N, y_{2}\right)$ & $\left(N, y_{3}\right)$ & $\left(N, y_{1}\right)$ & $\left(N, y_{5}\right)$ & $\left(N \backslash\{1\}, y_{4}\right)$ & $\left(N \backslash\{2\}, y_{4}\right)$ \\
161 & {$[\varnothing]_{1}$} & {$[\varnothing]_{2}$} & {$[\varnothing]_{3}$} & {$[\varnothing]_{4}$} & {$[\varnothing]_{5}$} & {$[\varnothing]_{6}$}
\end{tabular}

First, plurality voting does not satisfy consistency since $f^{P}(R)=\left(N \backslash\{6\}, y_{4}\right)$ but at the same time $f^{P}\left(R_{\mid N \backslash\{6\}}\right)=\left(N \backslash\{6\}, y_{1}\right)$.

\footnotetext{
${ }^{8}$ To obtain the vote of an agent we have to use information contained in the full profile, but since we are not considering the strategic aspect of preference revelation, this is not an issue.
} 
Second, the Borda rule does not satisfy consistency since $f^{B}(R)=\left(N \backslash\{6\}, y_{4}\right)$ but $f^{B}\left(R_{\mid N \backslash\{6\}}\right)=\left(N \backslash\{6\}, y_{1}\right)$. To see that, in the Borda rule the first alternative receives 160 points, the second 159 , the third 158 and so on, and each alternative in the class $[\varnothing]_{i}$ receives zero points (since all alternatives in the indifference class $[\varnothing]_{i}$ receive zero points). The alternatives ranked higher than $\left(N \backslash\{6\}, y_{1}\right)$ by some agent in $N \backslash\{6\}$ at $R$ are: $\left(N, y_{1}\right),\left(N, y_{2}\right)$, $\left(N, y_{3}\right),\left(N \backslash\{6\}, y_{4}\right),\left(N \backslash\{1\}, y_{4}\right),\left(N \backslash\{2\}, y_{4}\right)$ and $\left(N \backslash\{3\}, y_{4}\right)$. Add to this list the alternatives $\left(N \backslash\{6\}, y_{1}\right)$ and $\left(N, y_{5}\right)$ and observe that the remaining alternatives receive less points than $\left(N \backslash\{6\}, y_{1}\right)$. The table below shows the number of points (or an upper bound) assigned at $R$ by each $i \in N$ (in the columns) to each alternative in the list and the total number of points (or an upper bound) they obtain (in the last column).

\begin{tabular}{c|ccccccc} 
at $R$ & 1 & 2 & 3 & 4 & 5 & 6 & Total \\
\hline$\left(N \backslash\{6\}, y_{4}\right)$ & 158 & 158 & 158 & 160 & 160 & 0 & 794 \\
$\left(N \backslash\{6\}, y_{1}\right)$ & 159 & 159 & 159 & 156 & 156 & 0 & 789 \\
$\left(N, y_{1}\right)$ & 160 & 2 & 1 & $\leq 155$ & $\leq 155$ & 156 & $\leq 629$ \\
$\left(N, y_{2}\right)$ & 1 & 160 & 2 & $\leq 155$ & $\leq 155$ & 157 & $\leq 630$ \\
$\left(N, y_{3}\right)$ & $\leq 157$ & 1 & 160 & 2 & $\leq 155$ & 158 & $\leq 633$ \\
$\left(N, y_{5}\right)$ & $\leq 157$ & $\leq 157$ & $\leq 157$ & 1 & 2 & 160 & $\leq 634$ \\
$\left(N \backslash\{1\}, y_{4}\right)$ & 0 & $\leq 157$ & $\leq 157$ & 159 & 159 & $\leq 155$ & $\leq 787$ \\
$\left(N \backslash\{2\}, y_{4}\right)$ & $\leq 157$ & 0 & $\leq 157$ & 158 & 158 & 1 & $\leq 631$ \\
$\left(N \backslash\{3\}, y_{4}\right)$ & 2 & $\leq 157$ & 0 & 157 & 157 & $\leq 155$ & $\leq 628$
\end{tabular}.

Hence, $f^{B}(R)=\left(N \backslash\{6\}, y_{4}\right)$.

Similarly, for each $i \in N \backslash\{6\}, \#\left\{(S, x) \mid(S, x)\left(P_{\mid N \backslash\{6\}}\right)_{i}[\varnothing]_{i}\right\}=2^{4} \times 5=80$ is the number of alternatives that at $R_{\mid N \backslash\{6\}}$ each agent $i \in N \backslash\{6\}$ ranks strictly above [ø] $i$; hence, in the Borda rule the first alternative receives 80 points, the second 79 and so on. The only alternative ranked higher than $\left(N \backslash\{6\}, y_{1}\right)$ by some agent at $R_{\mid N \backslash\{6\}}$ is $\left(N \backslash\{6\}, y_{4}\right)$. The remaining alternatives receive less points that $\left(N \backslash\{6\}, y_{1}\right)$. The table below shows the number of points assigned by each $i \in N \backslash\{6\}$ (in the columns) to the two alternatives with the two highest number of total points received at $R_{\mid N \backslash\{6\}}$.

\begin{tabular}{c|cccccc} 
at $R_{\mid N \backslash\{6\}}$ & 1 & 2 & 3 & 4 & 5 & Total \\
\hline$\left(N \backslash\{6\}, y_{4}\right)$ & 79 & 79 & 79 & 80 & 80 & 397 \\
$\left(N \backslash\{6\}, y_{1}\right)$ & 80 & 80 & 80 & 79 & 79 & 398
\end{tabular}.


Hence, $f^{B}\left(R_{\mid N \backslash\{6\}}\right)=\left(N \backslash\{6\}, y_{1}\right)$. Thus, the Borda rule is not consistent.

Approval voting satisfies not only consistency (and internal stability by definition) but also other desirable properties. Before stating this result we need to specify a tie-breaking rule, to be used whenever two or more alternatives obtain the highest number of votes. The idea is simple. Suppose that several alternatives obtain the largest number of votes. We choose the alternative with coalition $S$ in $2^{N}$ ranked higher following an specified monotonic and complete order $\rho$ over coalitions. Since the order is monotonic, we choose an alternative associated with a coalition $S$ which is not contained in other coalition $T$ associated with an alternative with the highest number of votes. If it is unique, we are done. Suppose not, then we still have several alternatives with the highest number of votes, but now all of them coincide in having the same coalition $S$ but together with a different outcome in $X$. We now choose the agent $i$ in $S$ ranked higher according to the order $\rho$. We select, among the alternatives with the highest number of votes having $S$ as the final society, the one preferred by agent $i$. We now define this tie-braking rule formally.

Let $\rho$ be a monotonic and complete order over $2^{N}$. Namely, for each pair $S, T \in 2^{N}$ such that $S \subsetneq T, T \rho S$. Observe that $N \rho S$ for all $S \neq N$. Denote by $f^{A V, \rho}$ the approval voting rule induced by $\rho$ as follows. Let $A^{\prime}=\left\{\left(S_{k}, x_{k}\right)\right\}_{k=1}^{K}$ be the set of alternatives that have received the largest number of votes according to approval voting at $R$. If $S_{k}=\varnothing$ for all $k \in\{1, \ldots, K\}$, select $(\varnothing, y)$ where $y$ is such that $(N, y) P_{i}(N, z)$ for all $z \neq y$ and $\{i\} \rho\{j\}$ for all $j \neq i$. Assume $S_{k} \neq \varnothing$ for some $k \in\{1, \ldots, K\}$. First select the final society $S \in\left\{S_{1}, \ldots, S_{K}\right\}$ ranked highest by $\rho$ and consider the subset of alternatives $\left\{\left(S_{k^{\prime}}, x_{k^{\prime}}\right) \in A^{\prime} \mid S_{k^{\prime}}=S\right\}$. Select again the agent $i \in S$ who is ranked highest by $\rho$ (as a singleton set) and choose finally as $f^{A V, \rho}(R)$ the alternative most preferred by $i$ among those in the family $\left\{\left(S_{k^{\prime}}, x_{k^{\prime}}\right) \in A^{\prime} \mid S_{k^{\prime}}=S\right\}$.

Proposition 1 below states that any approval voting $f^{A V, \rho}$ is internally stable, consistent, and additionally satisfies other desirable properties.

Proposition 1 Let $\rho$ be a monotonic and complete order over $2^{N}$. Then, the approval voting $f^{A V, \rho}$ satisfies internal stability, consistency, efficiency and neutrality. Moreover, in the subdomain of profiles where the tie-breaking rule is not applied, $f^{A, \rho}$ satisfies anonymity.

Proof Observe that if $(S, x)$ is approved by $i$, then $i \in S$. This fact will be repeatedly used in the proof.

- Internal stability. By definition, $f^{A, \rho}$ is internally stable. 
- Consistency. Let $R \in \mathcal{R}$ be arbitrary and let $f^{A V, \rho}(R)=(S, x)$ be such that $S \neq \varnothing$. The set of agents approving $(S, x)$ at $R$ coincides with the set of agents approving $(S, x)$ at $R_{\mid f_{N}^{A, \rho}(R)}$. Hence, $f^{A V, \rho}\left(R_{\mid f_{N}^{A, \rho}(R)}\right)=f^{A V, \rho}(R)$ and thus, $f^{A V, \rho}$ is consistent.

- Efficiency. Suppose otherwise; namely, there exist $R \in \mathcal{R}$ and $(S, x) \in A$ such that $(S, x) R_{i} f^{A V, \rho}(R)$ for all $i \in N$ and there exists $j \in N$ such that $(S, x) P_{j} f^{A V, \rho}(R)$. Hence, $(S, x) \neq f^{A V, \rho}(R)$. Assume first that $f_{N}^{A V, \rho}(R)=\varnothing$, which implies that $f^{A V, \rho}(R)$ did not receive any vote and $S \neq \varnothing$. By (P.2), $(S, x) P_{j} f^{A V, \rho}(R)$ for all $j \in S$ and $(S, x) I_{j} f^{A V, \rho}(R)$ for all $j \notin S$. But this means that $(S, x)$ received more votes than $f^{A V, \rho}(R)$, a contradiction. Assume now that $f_{N}^{A V, \rho}(R) \neq \varnothing$ and let $i \in f_{N}^{A V, \rho}(R)$. Since $f^{A V, \rho}$ satisfies internal stability, $f^{A V, \rho}(R) P_{i}[\varnothing]_{i}$. Hence, $i \in S$ and, by the contradiction hypothesis and (P.2), $(S, x) P_{i} f^{A V, \rho}(R)$. We consider two cases. First, $f_{N}^{A V, \rho}(R) \varsubsetneqq S$. Since for each $j \in S \backslash f_{N}^{A V, \rho}(R), f^{A V, \rho}(R) \in[\varnothing]_{j}$ and $(S, x) R_{i} f^{A V, \rho}(R)$ for all $i$, it follows that $(S, x)$ has received more votes than $f^{A V, \rho}(R)$, a contradiction. Second, $f_{N}^{A V, \rho}(R)=$ $S$. Then, $f^{A, V \rho}(R)=(S, y)$ with $y \neq x$ and all agents in $S$ have approved both, $(S, x)$ and $(S, y)$. This means that the tie-breaking rule $\rho$ has been used to select $f^{A V, \rho}(R)$, implying that there exists $i \in S$ such that $f^{A V, \rho}(R) P_{i}(S, x)$ which is a contradiction.

- Neutrality. Let $R \in \mathcal{R}$ and $\sigma \in \Sigma$. Observe that the number of agents approving $(S, x)$ at $R$ coincides with the number of agents approving $(S, \sigma(x))$ at $R^{\sigma}$. We consider two cases. First, $f^{A V, \rho}(R)$ has been approved at $R$ by more agents than any other alternative. Hence, $\left(f_{N}^{A V, \rho}(R), \sigma\left(f_{X}^{A V, \rho}(R)\right)\right)$ has been approved at $R^{\sigma}$ by more agents than any other alternative, implying that $f^{A V, \rho}\left(R^{\sigma}\right)=f^{A V, \rho}(R)$. Second, it is necessary to apply $\rho$ to select $f^{A V, \rho}(R)$. Let $\left\{\left(S_{k}, x_{k}\right)\right\}_{k=1}^{K}$ be the set of alternatives receiving the largest number of votes at $R$. Thus, $\left\{\left(S_{k}, \sigma\left(x_{k}\right)\right)\right\}_{k=1}^{K}$ is the set of alternatives receiving the largest number of votes at $R^{\sigma}$. Hence,

$$
f_{N}^{A V, \rho}\left(R^{\sigma}\right)=f_{N}^{A V, \rho}(R)
$$

Now, let $i \in f_{N}^{A V, \rho}(R)$ be the agent with the highest ranking, among singleton sets, according to $\rho$ and let $i^{\prime} \in f_{N}^{A V, \rho}\left(R^{\sigma}\right)$ be the agent with the highest ranking, among singleton sets, according to $\rho$. By $(1) i^{\prime}=i$. Thus, $f_{X}^{A V, \rho}\left(R^{\sigma}\right)=\sigma\left(f_{X}^{A V, \rho}(R)\right)$, which together with (1) implies that $f^{A V, \rho}\left(R^{\sigma}\right)=\left(f_{N}^{A V, \rho}(R), \sigma\left(f_{X}^{A V, \rho}(R)\right)\right)$. 
- Anonymity on the subdomain of profiles where the tie-breaking rule is not applied. Let $R$ be one of such profiles. Then, $f^{A V, \rho}(R)$ has been approved by more agents than any other alternative. Observe that the number of agents approving any $(S, x)$ at $R$ coincides with the number of agents approving $(\pi(S), x)$ at $R^{\pi}$. Thus, $\left(\pi\left(f_{N}^{A V, \rho}(R)\right), f_{X}^{A V, \rho}(R)\right)$ has been approved at $R^{\pi}$ by more agents that any other alternative. Hence, $f^{A V, \rho}\left(R^{\pi}\right)=$ $\left(\pi\left(f^{A V, \rho}(R)\right), f_{X}^{A V, \rho}(R)\right)$, which means that $f^{A V, \rho}$ satisfies anonymity at $R$.

Remark 1 Proposition 1 also holds with other tie-breaking rules. For instance consider an order $\pi$ of the set of agents and assume that agents choose between the set of alternatives $A^{\prime}=\left\{\left(S_{k}, x_{k}\right)\right\}_{k=1}^{K}$ with the largest number of votes according to approval voting at $R$ following such order. Namely, if $S_{k}=\varnothing$ for all $k$, select $(\varnothing, y)$ where $y$ is such that $(N, y) P_{\pi_{1}}(N, z)$ for all $z \neq y$. Assume $S_{k} \neq \varnothing$ for some $k$. Let $\left(S_{1}, x_{1}\right)$ in the best indifference class of agent $\pi_{1}$ in $A^{\prime}$. If $\pi_{1} \in S_{1}$, set $f^{A V, \pi}(R)=\left(S_{1}, x_{1}\right)$. Otherwise, look for any alternative $\left(S_{2}, x_{2}\right)$ in the best indifference class of agent $\pi_{2}$ in $A^{\prime \prime}=\left\{\left(S_{k}, x_{k}\right) \in A^{\prime}: \pi_{1} \notin S_{k}\right\}$. If $\pi_{2} \in S_{2}$, set $f^{A V, \pi}(R)$. Otherwise, proceed similarly until the $n$-th step.

We end this note by applying the Condorcet winner to our setting. First, we recall the definition of the Condorcet winner in the classical setting. Fix a profile $P$ of strict preferences over $X$ and let $x, y \in X$ be such that $x \neq y$. We say that $x$ beats $y$ if the number of agents preferring $x$ to $y$ is strictly larger than the number of agents preferring $y$ to $x$. We say that $x$ is a Condorcet winner at $P$ if there is no $y$ that beats $x$. There are profiles at which no Condorcet winner exists and others at which there are several Condorcet winners. Thus, the Condorcet winner is not a rule.

We adapt the notion of a Condorcet winner to our setting as we have already did for the previous two classes of rules. In order to ensure that the chosen alternative satisfies internal stability at $R$ we only consider votes for unanimously internally stable alternatives at $R$. When several Condorcet winners exist we apply the tie-breaking (using a monotonic and complete order $\rho$ ) used to define approval voting.

We say that a profile $R \in \mathcal{R}$ is resolute if there exists $a \in A$ such that $a$ beats $a^{\prime}$ for all $a^{\prime} \neq a$. Thus, the Condorcet winner selects $a$ at $R$. Let $f^{C, \rho}(R)$ denote the Condorcet winner (if any) at $R$. If $R \in \mathcal{R}$ is resolute, then $f^{C, \rho}(R)$ is independent of $\rho$ and $\left|f^{C, \rho}(R)\right|=1$. Proposition 2 states that the Condorcet winner at resolute profiles satisfies the same properties as Approval voting, at such profiles. 
Proposition 2 Let $R$ be a resolute profile. Then, $f^{C, \rho}(R)$ satisfies internal stability, consistency, efficiency, neutrality and anonymity at $R$.

Proof Fix a resolute profile $R$ and set $f^{C, \rho}(R)=(S, x)$. We show that $f^{C, \rho}(R)$ satisfies the properties at $R$.

- Internal stability. By definition, $f^{C, \rho}(R)$ satisfies internal stability at $R$.

- Consistency. We prove that $f^{C, \rho}\left(R_{\mid S}\right)=(S, x)$ by showing that at $R_{\mid S},(S, x)$ beats $(T, y)$ for all $(T, y) \neq(S, x)$ with $T \subset S$. Let $(T, y)$ be an alternative with the above properties. Since $(S, x)$ beats $(T, y)$ at $R$, the number of agents in $N$ preferring $(S, x)$ to $(T, y)$ is strictly larger than the number of agents in $N$ preferring $(T, y)$ to $(S, x)$. Moreover, each agent in $N \backslash S$ is indifferent between $(S, x)$ and $(T, y)$. Thus the number of agents in $S$ preferring $(S, x)$ to $(T, y)$ (or $(T, y)$ to $(S, x)$ ) coincides with the number of agents in $N$ preferring $(S, x)$ to $(T, y)$ (or $(T, y)$ to $(S, x)$ ). Hence, $(S, x)$ beats $(T, y)$ at $R_{\mid S}$, implying that $f^{C, \rho}\left(R_{\mid S}\right)=(S, x)$.

- Efficiency. Suppose otherwise; in particular, there must exist $(T, y)$ such that $(T, y) R_{i}(S, x)$ for all $i \in N$ and $(S, x) \neq(T, y)$. Let $i \in S$. Since $(S, x)$ satisfies internal stability at $R$, $(S, x) P_{i}[\varnothing]_{i}$. Hence, $i \in T$ and $(T, y) P_{i}(S, x)$. Each agent in $N \backslash T$ is indifferent between $(S, x)$ and $(T, y)$. Thus $(T, y)$ beats $(S, x)$, which contradicts that $f^{C, \rho}(R)=(S, x)$.

- Neutrality. Observe that for each $(T, y) \neq(S, x),(S, \sigma(x))$ beats $(T, \sigma(y))$ at $R^{\sigma}$. Hence, $f^{C, \rho}\left(R^{\sigma}\right)=(S, \sigma(x))$, which means that $f^{C, \rho}$ satisfies neutrality at $R$.

- Anonymity. Observe that for each $(T, y) \neq(S, x),(\pi(S), x)$ beats $(\pi(T), y)$ at $R^{\pi}$. Hence, $f^{C, \rho}\left(R^{\pi}\right)=(\pi(S), x)$, which means that $f^{C, \rho}$ satisfies anonymity at $R$.

Nevertheless, for non-resolute profiles the Condorcet winner, even when it is unique, may not satisfy consistency. To see that, consider the following example.

Example 3 Let $N=\{1,2,3,4,5\}$ and $X=\left\{y_{1}, y_{2}\right\}$ be respectively the set of agents and outcomes and let $\rho$ be any monotonic and complete order over $2^{N}$ satisfying $\{1\} \rho\{2\} \rho\{3\} \rho\{4\} \rho\{5\}$. Consider any profile $R$ satisfying the following properties, where the first column indicates the 
rank of each of the five preference relations.

\begin{tabular}{l|ccccc} 
Rank & $R_{1}$ & $R_{2}$ & $R_{3}$ & $R_{4}$ & $R_{5}$ \\
\hline First & $\left(N \backslash\{5\}, y_{1}\right)$ & $\left(N \backslash\{5\}, y_{1}\right)$ & $\left(N \backslash\{5\}, y_{2}\right)$ & $\left(N \backslash\{5\}, y_{2}\right)$ & $\left(N, y_{1}\right)$ \\
Second & $\left(N \backslash\{5\}, y_{2}\right)$ & $\left(N \backslash\{5\}, y_{2}\right)$ & $\left(N, y_{1}\right)$ & $\left(N, y_{1}\right)$ & {$[\varnothing]_{5}$} \\
Third & $\left(N, y_{1}\right)$ & $\left(N, y_{1}\right)$ & $\left(N \backslash\{5\}, y_{1}\right)$ & $\left(N \backslash\{5\}, y_{1}\right)$ & \\
Fourth & {$[\varnothing]_{1}$} & {$[\varnothing]_{2}$} & {$[\varnothing]_{3}$} & {$[\varnothing]_{4}$} &
\end{tabular}

The only internally stable alternatives are $\left(N \backslash\{5\}, y_{1}\right),\left(N \backslash\{5\}, y_{2}\right)$, and $\left(N, y_{1}\right)$. At $R$, $\left(N \backslash\{5\}, y_{1}\right)$ ties with $\left(N \backslash\{5\}, y_{2}\right)$ (so they do not beat each other), $\left(N \backslash\{5\}, y_{2}\right)$ beats $\left(N, y_{1}\right)$ and $\left(N, y_{1}\right)$ beats $\left(N \backslash\{5\}, y_{1}\right)$. Therefore, $R$ is not resolute because $\left(N \backslash\{5\}, y_{2}\right)$ does not beat $\left(N \backslash\{5\}, y_{1}\right)$. Since $\left(N \backslash\{5\}, y_{2}\right)$ is the unique Condorcet winner (no alternative beats it), $f^{C, \rho}(R)=\left(N \backslash\{5\}, y_{2}\right)$. To check for consistency of $f$, consider the subprofile $R_{\mid N \backslash\{5\}}$ given by

\begin{tabular}{l|cccc} 
& $\left(R_{\mid N \backslash\{5\}}\right)_{1}$ & $\left(R_{\mid N \backslash\{5\}}\right)_{2}$ & $\left(R_{\mid N \backslash\{5\}}\right)_{3}$ & $\left(R_{\mid N \backslash\{5\}}\right)_{4}$ \\
\hline First & $\left(N \backslash\{5\}, y_{1}\right)$ & $\left(N \backslash\{5\}, y_{1}\right)$ & $\left(N \backslash\{5\}, y_{2}\right)$ & $\left(N \backslash\{5\}, y_{2}\right)$ \\
Second & $\left(N \backslash\{5\}, y_{2}\right)$ & $\left(N \backslash\{5\}, y_{2}\right)$ & $\left(N \backslash\{5\}, y_{1}\right)$ & $\left(N \backslash\{5\}, y_{1}\right)$ \\
Third & {$[\varnothing]_{1}$} & {$[\varnothing]_{2}$} & {$[\varnothing]_{3}$} & {$[\varnothing]_{4}$}
\end{tabular}.

At $R_{\mid N \backslash\{5\}},\left(N \backslash\{5\}, y_{1}\right)$ ties with $\left(N \backslash\{5\}, y_{2}\right)$ and they beat all other alternatives. Hence, the two are Condorcet winners at $R_{\mid N \backslash\{5\}}$. Thus, applying the tie-breaking rule $\rho$, and since 1 prefers $\left(N \backslash\{5\}, y_{1}\right)$ to $\left(N \backslash\{5\}, y_{2}\right)$, we have that $f^{C, \rho}\left(R_{\mid N \backslash\{5\}}\right)=\left(N \backslash\{5\}, y_{1}\right)$, which means that $f^{C, \rho}$ does not satisfy consistency.

Using arguments similar to those used in the proof of Proposition 1 we can prove that the scoring methods satisfy efficiency and neutrality in the general domain and anonymity when no tie breaking rule is used. We now present a table summarizing our main findings.

\begin{tabular}{|c|c|c|c|c|}
\hline Properties & Approval & Condorcet & Scoring & Serial Dictator \\
\hline Internal Stability & General domain & Resolute & General domain & \\
\hline Consistency & General domain & Resolute & & General domain \\
\hline Efficiency & General domain & Resolute & General domain & General domain \\
\hline Neutrality & General domain & Resolute & General domain & \\
\hline Anonymity & No tie breaking & Resolute & No tie breaking & \\
\hline
\end{tabular}




\section{$5 \quad$ Final remarks}

Before finishing the paper two remarks related to our domain restriction are in order.

First, our main results do not hold without (P.1) because approval voting and the Condorcet winner are not consistent at profiles that do not satisfy (P.1). For instance, consider the example where $N=\{1,2,3,4,5\}$ and $X=\{x, y\}$ are respectively the set of agents and outcomes, and let $P=\left(R_{1}, R_{2}, R_{3}, R_{4}, R_{5}\right)$ be the following preference profile:

\begin{tabular}{c|ccccc} 
Rank & $R_{1}$ & $R_{2}$ & $R_{3}$ & $R_{4}$ & $R_{5}$ \\
\hline First & $(\{123\}, x)$ & $(\{123\}, x)$ & $(\{123\}, y)$ & $(\{123\}, y)$ & $(\{123\}, y)$ \\
Second & $(\{123\}, y)$ & $(\{123\}, y)$ & $(\{123\}, x)$ & $(\{123\}, x)$ & $(\{123\}, x)$ \\
Third & $(\varnothing, x)$ & $(\varnothing, x)$ & $(\varnothing, x)$ & $(\varnothing, x)$ & $(\varnothing, x)$
\end{tabular}.

Let $\rho$ be arbitrary. Since $f^{A, \rho}(R)=(\{123\}, y)$ but $f^{A, \rho}\left(R_{\mid\{1,2,3\}}\right)=(\{123\}, x)$ approval voting is not consistent and hence Proposition 1 does not hold if agents' preferences do not satisfy (P.1). Since $f^{C, \rho}(R)=(\{123\}, y)$ but $f^{C, \rho}\left(R_{\mid\{1,2,3\}}\right)=(\{123\}, x)$ the Condorcet winner is not consistent and hence Proposition 2 does not hold if agents' preferences do not satisfy (P.1).

Second, our domain of preferences satisfying conditions (P.1) and (P.2), yet restrictive, is still very large. And hence, one may ask about the possible extension of our results to meaningful subdomains for specific problems. First, for the case of an excludable public good (in a linearly ordered set $X$ of outcomes) when agents have single-peaked preferences over $X$ and also care about the size of the set of its users, as in Jackson and Nicolò (2004). Second, as in Berga, Bergantiños, Massó and Neme (2004), where a set of founders of a society are considering the possibility of admitting new members from a given set of candidates, and founders preferences on final societies are separable (or additive). Third, assume agent $i$ has $(S, x)$ as top-ranked and $i \in S$. If $i \in T$, then $(T, x)$ should be ranked higher than $(T \backslash\{i\}, x)$; that is, $(T, x)$ "lies" somehow between $(S, x)$ and $(T \backslash\{i\}, x)$, and since $(S, x)$ is the top-ranked alternative, agent $i$ should prefer the alternative that is "closer" to the top. Each of those preference domains will require a specific analysis of the possibilities of designing individually rational and consistent rules, together with other desirable properties. However, we leave this analysis for further research. 


\section{References}

[1] Barberà, S., M. Maschler and J. Shalev (2001): "Voting for voters: a model of electoral evolution," Games and Economic Behavior 37, 40-78.

[2] Barberà, S. and A. Perea (2002): "Supporting others and the evolution of influence," Journal of Economic Dynamics \& Control 26, 2051-2092.

[3] Berga, D., G. Bergantiños, J. Massó and A. Neme (2004): "Stability and voting by committees with exit," Social Choice and Welfare 23, 229-247.

[4] Berga, D., G. Bergantiños, J. Massó and A. Neme (2006): "On exiting after voting," International Journal of Game Theory 34, 33-54.

[5] Berga, D., G. Bergantiños, J. Massó and A. Neme (2007): "An undominated Nash equilibrium for voting by committees with exit," Mathematical Social Sciences 54, 152-175.

[6] Bergantiños, G., J. Massó and A. Neme (2015): "The division problem under constraints," Games and Economic Behavior 89, 56-77.

[7] Bergantiños, G., J. Massó and A. Neme (2017): "On societies choosing social outcomes, and their memberships: strategy-proofness," Social Choice and Welfare 48, 857-875.

[8] Jackson, M. and A. Nicolò (2004): "The strategy-proof provision of public goods under congestion and crowding preferences," Journal of Economic Theory 115, 278-308.

[9] Nizamogullari, D. and İ. Özkal-Sanver (2014): "Characterization of the core in full domain marriage problems," Mathematical Social Sciences 69, 34-42.

[10] Nizamogullari, D. and İ. Özkal-Sanver (2015): "Consistent enlargements of the core in roommate problems," Theory \& Decision 79, 217-225.

[11] Özkal-Sanver, İ. (2013): "Minimal conversely consistent extension of the men-optimal solution," Social Choice and Welfare 40, 89-99.

[12] Sasaki, H. and M. Toda (1992): "Consistency and characterization of the core of two-sided matching problems," Journal of Economic Theory 56, 218-237. 
[13] Roberts, K. (1999): "Dynamic voting in clubs," mimeo, London School of Economics.

[14] Sobel, J. (2000): "A model of declining standard," International Economic Review 41, 295-303.

[15] Thomson, W. (1994): "Consistent solutions to the problem of fair division when preferences are single-peaked," Journal of Economic Theory 63, 219-245.

[16] Thomson, W. (2007): "On the existence of consistent rules to resolve conflicting claims: a geometric approach," Review of Economic Design 11, 225-251. 